\title{
Fatores de Risco para Infecção Pós-histerectomia Total Abdominal
}

\author{
Risk Factors for Infection after Total Abdominal Hysterectomy \\ Melania Maria Ramos de Amorim, Luiz Carlos Santos, Vilma Guimarães
}

\section{RESUMO}

\begin{abstract}
Objetivos: determinar os principais fatores associados à ocorrência de infecção do sítio cirúrgico em pacientes submetidas a histerectomia total abdominal (HTA) no Instituto Materno - Infantil de Pernambuco (IMIP).

Métodos: realizou-se um estudo de corte transversal incluindo todas as pacientes submetidas a histerectomia total abdominal no IMIP no periodo de janeiro de 1995 a dezembro de 1998, desde que tivessem retornado no $7^{\circ}$ e no $30^{\circ}$ dia pós-operatório para controle de infecção $(n=$ 414). A freqüência de infecção do sitio cirúrgico (definida pelos critérios do CDC, 1998) foi de 10\% (42 casos). Calculou-se o risco de prevalência (RP) de infecção do sítio cirúrgico e seu intervalo de confiança (IC) a 95\% para as seguintes variáveis: idade, obesidade, hipertensão, diabetes, doença maligna, tipo de incisão, tempo cirúrgico e antibioticoprofilaxia. Realizou-se análise de regressão logística múltipla para determinação do risco ajustado de infecção.

Resultados: encontrou-se aumento significativo do risco de infecção do sítio cirúrgico para as seguintes variáveis: idade $\geq 60$ anos $(R P=2,39 ; I C-95 \%=1,15-4,94)$, obesidade $(R P=3,2$; IC$95 \%=1,83-5,59)$, duração da cirurgia $>2$ horas $(R P=2,36 ;$ IC-95\% $=1,32-4,21)$ e associação com diabetes $(R P=6,0 ; I C-95 \%=3,41-10,57)$. Por outro lado, o risco de infecção esteve significativamente diminuido quando utilizou-se antibiótico profilático $(R P=0,38$; IC-95\% =0,210,68). Não se encontrou associação estatisticamente significativa de infecção com o tipo de incisão, a indicação da cirurgia por patologia maligna e a presença de hipertensão.

Conclusões: os fatores associados a risco aumentado de infecção do sítio cirúrgico pós-HTA no IMIP foram: idade >60 anos, obesidade, diabetes e duração da cirurgia $>2$ horas. A antibioticoprofilaxia apresentou efeito protetor, com diminuição do risco de infecção.
\end{abstract}

PALAVRAS-CHAVE: Histerectomia. Infecção hospitalar. Complicações pós-operatórias.

\section{Introdução}

A histerectomia constitui atualmente uma das cirurgias mais realizadas em todo o mundo, a maioria delas por via abdominal ${ }^{1,2}$. Nos Estados Unidos, representa a cirurgia mais comum em mulheres depois da cesariana, realizandose em torno de 600.000 histerectomias a cada ano. Acredita-se que mais de 20 milhões de americanas já foram submetidas a este procedimen$t^{1,2}$. As indicações mais freqüentes são as doenças benignas (leiomiomatose uterina, endome-

Instituto Materno-Infantil de Pernambuco (IMIP)

Prêmio FEBRASGO de Ginecologia no $48^{\circ}$ Congresso Brasileiro de Ginecologia e Obstetrícia; Goiânia, novembro de 1999.

Correspondência: Melania Maria Ramos de Amorim

Coordenação de Ensino do IMIP

Rua dos Coelhos, 300 - Ilha do Leite

50070-550 - Recife - PE

e-mail: melania@interway.com.br triose, hiperplasias), ao passo que as doenças malignas representam em torno de $10 \%$ das indicações ${ }^{1}$.

Apesar de ter se tornado uma cirurgia relativamente segura na atualidade, a morbidade associada à histerectomia não é desprezivel ${ }^{1,3,4}$. $\mathrm{O}$ risco de mortalidade é considerado baixo, em torno de 1 a 2 para cada 1000 intervenções, conseqüência direta dos progressos no tratamento das doenças clínicas associadas, dos cuidados pré e pós-operatórios, do uso adequado de hemotransfusão e antibióticos, bem como dos avanços nas técnicas anestésicas ${ }^{1}$. No entanto, as complicações pós-operatórias são ainda importantes, estimando-se que até $25 \%$ das histerectomias vaginais e $50 \%$ das histerectomias abdominais cursem com algum tipo de complicação ${ }^{1-4}$.

A morbidade infecciosa representa uma 
das complicações mais importantes associadas à histerectomia, ocorrendo em freqüência variável de serviço para serviço ${ }^{5-8}$. O risco é maior nas histerectomias abdominais (variando de 3,9-50\%) em relação às vaginais ${ }^{1}$, o que tem sido atribuído à não-abertura da parede abdominal na via vaginal e, sobretudo, à maior eficácia da antibioticoterapia profilática nas operações vaginais s $^{3,5,7}$.

Entre as infecções pós-operatórias, a infecção do sítio cirúrgico desponta como uma das maiores causas de morbidade e mortalidade póshisterectomia ${ }^{3,4,8}$. O termo foi definido em 1998 pelo "Centers for Disease Control" (CDC), substituindo o antigo termo "infecção de ferida pósoperatória", e compreende 3 subgrupos: infecção superficial (interessando pele e tecido celular subcutâneo), infecção profunda (abscessos no nivel subaponeurótico ou submuscular) e infecção de órgãos e espaços (infecção/abscessos cavitários) $)^{9}$.

Diversos fatores de risco têm sido apontados para o desenvolvimento de infecção pós-operatória, como o baixo nível socioeconômico, a idade avançada, obesidade, diabetes, neoplasias malignas, tempo cirúrgico aumentado, hospitalização prolongada e uso de drenos ${ }^{1,4,8}$. Vaginose bacteriana e tricomoníase genital também estão associadas a aumento do risco de infecção em cirurgias ginecológicas, particularmente na histerectomia abdominal ${ }^{1,10}$.

O conhecimento e a adequada identificação desses fatores de risco assumem especial interesse no capítulo de prevenção da morbidade pós-operatória, inclusive no sentido de definir a utilização ou não de antibioticoprofilaxia. A efetividade desta última na histerectomia abdominal é ainda controversa, conquanto seja irrefutável sua indicação nas histerectomias vaginais s $^{5,8,11}$. Recomenda-se portanto que sua indicação se paute pela freqüência de infecção do sítio cirúrgico nos diversos serviços, devendo ser utilizada sempre que esta for superior a $10 \%{ }^{5}$.

Realizou-se o presente estudo com o objetivo de determinar os principais fatores de risco associados à infecção do sítio cirúrgico em pacientes submetidas a histerectomia total abdominal no Centro de Atenção à Mulher do IMIP.

\section{Métodos}

Realizou-se um estudo de corte transversal incluindo todas as pacientes submetidas a histerectomia total abdominal (HTA) no IMIP no período de janeiro de 1995 a dezembro de 1998, desde que tivessem retornado no $7^{\circ}$ e no $30^{\circ}$ dia pós-operatório para revisão cirúrgica e controle da infecção. Coletaram-se os dados a partir dos prontuários médicos, de janeiro a março de 1999. Excluiram-se as pacientes que realizaram outros procedimentos cirúrgicos concomitantes, como por exemplo cura de incontinência urinária de esforço (via alta ou baixa), os casos de histerectomia radical (Wertheim-Meigs) e as pacientes que não retornaram para revisão no periodo mencionado. Ao final, o número total de pacientes estudadas foi de 414 , das quais $42(10 \%)$ apresentaram infecção do sítio cirúrgico. As principais características dessas pacientes são apresentadas na Tabela 1 .

Tabela 1 - Características gerais das pacientes submetidas a histerectomia total abdominal no IMIP.

\begin{tabular}{lccrr}
\hline Característica & Média \pm DP & Variação & $\%$ & $\mathbf{n}$ \\
\hline Idade (anos) & $45,4 \pm 9,8$ & $30-90$ & & \\
Paridade (mediana) & 3 & $0-22$ & & \\
Obesidade & & & 20,5 & 80 \\
Diabetes & & & 4,3 & 18 \\
Hipertensão & & & 18,1 & 75 \\
Doença maligna & & 4,3 & 18 \\
Duração da cirurgia & $116 \pm 31,9$ & $40-210$ & & \\
(minutos) & & & & \\
Tipo de incisão & & & 23,0 & 95 \\
- mediana & & & 77,0 & 319 \\
- Pfannenstiel & & & & \\
Antibioticoprofilaxia & & & 84,0 & 348 \\
Infecção & & 10,0 & 42 \\
\hline DP= desvio padrão & & & &
\end{tabular}

Considerou-se como variável dependente a ocorrência ou não de infecção do sítio cirúrgico, e como variáveis independentes os seguintes fatores: idade, indicação cirúrgica, obesidade, doenças associadas (hipertensão e diabetes), tipo de incisão, duração da cirurgia e antibioticoprofilaxia.

Definiu-se infecção do sítio cirúrgico (ISS) de acordo com os critérios do "Centers for Disease Control" - CDC $(1998)^{9}$, categorizando-se esta variável como presente ou ausente. Quando presente, foi classificada, ainda de acordo com os critérios do CDC, em superficial (envolvendo pele e tecido subcutâneo), profunda (aponeurose e músculos) e de órgãos e espaços ${ }^{1}$.

Considerou-se a idade (em anos) como variável contínua, ao passo que se definiu obesidade como um índice de massa corpórea (peso/ 
altura $^{2}$ ) superior a 27,5. Dividiram-se as indicações da cirurgia em duas categorias: por doença benigna (mioma, adenomiose, hiperplasia endometrial, etc.) ou maligna (câncer de corpo uterino ou de ovário). Os critérios diagnósticos para diabetes mellitus foram uma glicemia de jejum de $126 \mathrm{mg} \%$ ou superior ou ainda um valor da curva glicêmica igual ou superior a $200 \mathrm{mg} \%$. Diagnosticou-se hipertensão arterial sistêmica crônica pela presença de níveis tensionais de 140 $\mathrm{mmHg}$ ou mais de pressão sistólica e/ou 90 mmHg ou mais de pressão diastólica, em pelo menos 2 aferições.

Definiu-se como antibioticoterapia profilática o uso de antibiótico iniciado no momento da indução anestésica e mantido no transoperatório, embora não no pós-operatório, com a finalidade de prevenir a infecção do sítio cirúrgico (CDC, $1998)^{9}$. Os esquemas utilizados foram: cefalotina 1 g EV (repetindo-se a cada hora até o final da cirurgia) ou cefazolina $1 \mathrm{~g} \mathrm{EV}$ (repetindo-se apenas se o tempo cirúrgico ultrapassasse 3 horas). Como no início do período estudado ainda não havia sido normatizado o uso de antibioticoprofilaxia para HTA no IMIP, parte das pacientes não fizeram uso, uma vez que a decisão ficava a cargo de cada cirurgião. A partir de 1997, passouse a utilizar a cefazolina rotineiramente para antibioticoterapia profilática.

Todos os procedimentos cirúrgicos foram realizados pelos médicos-residentes de segundo ou terceiro ano, auxiliados pelos preceptores responsáveis, em geral sob bloqueio anestésico (raqui ou peridural), embora aproximadamente $5 \%$ das pacientes tenham sido submetidas a anestesia geral $(\mathrm{n}=20)$. O tipo de incisão de parede empregado foi preferencialmente à Pfannenstiel, embora incisão mediana infraumbilical tenha sido realizada em $23 \%$ dos casos $(\mathrm{n}=95)$. A histerectomia total abdominal com ou sem anexectomia foi realizada seguindo a técnica preconizada por Richardson e descrita no livro de Ginecologia Cirúrgica de Te Linde $^{1}$, com ligeiras modificações de acordo com a preferência do cirurgião. Quando não havia indicação de anexectomia, mesmo assim procedia-se à salpingectomia bilateral, conforme rotina do serviço. Caso a evolução fosse satisfatória, as pacientes tinham alta hospitalar no $3^{\circ}$ dia pós-operatório (DPO), com orientação de retorno no $7^{\circ}$ e no $30^{\circ}$ dia ao ambulatório de revisão cirúrgica.

Realizou-se a análise estatística nos programas Epi-Info 6.04b e SPSS 6.0 para Windows, utilizando-se médias e seus desvios-padrão, distribuição de freqüência e, na análise bivariada, os testes $\chi^{2}$ de associação (Pearson) e exato de
Fisher, a um nivel de significância de 5\%. Calculou-se o risco de prevalência (RP) de infecção do sítio cirúrgico para as diversas variáveis independentes, determinando-se os limites de seu intervalo de confiança a 95\% (IC-95\%). Regressão logística múltipla foi utilizada para determinação do risco de prevalência ajustado, em um modelo em que entraram a variável dependente (infecção do sitio cirúrgico) e as variáveis independentes (idade, indicação cirúrgica, obesidade, doenças associadas, tipo de incisão, duração da cirurgia e antibioticoprofilaxia), recodificadas binariamente $(1=\operatorname{sim} / 2=$ não). Essa etapa da análise foi realizada em SPSS para Windows, utilizando-se o comando Forward: Conditional.

\section{Resultados}

Considerando-se a associação entre idade e infecção do sítio cirúrgico, verificou-se um RP de $2,4(\mathrm{IC}-95 \%=1,2-4,9)$ para as pacientes com 60 anos ou mais. A obesidade, por sua vez, apresentou um risco 3,2 vezes maior de infecção (IC$95 \%=1,8-5,6)$. Esse risco também foi elevado na presença de diabetes $(\mathrm{RP}=6,0$, IC-95\% $=3,4-$ $10,6)$, permanecendo inalterado entre os casos de hipertensão, que não apresentaram associação significativa com infecção. Também não houve associação entre presença de doença maligna como indicação cirúrgica e infecção, permanecendo o risco inalterado (Tabela 2).

O uso de antibioticoterapia profilática esteve associado a significativa redução do risco de infecção do sítio cirúrgico, em torno de $60 \%$; verificou-se um risco de prevalência de $0,4(0,2-$ $0,7)$. Não houve associação entre o tipo de incisão (mediana ou Pfannenstiel) com a presença de infecção, porém os casos de abscesso subaponeurótico (ISS profunda) só foram encontrados quando se realizou incisão à Pfannenstiel ( $\mathrm{n}=15$ ). Quanto ao tempo cirúrgico, duração maior que 2 horas associou-se a significativo aumento do risco de infecção ( $R P=2,4$, IC-95\% = 1,3-4,2) (Tabela 2).

$\mathrm{Na}$ análise de regressão logística múltipla (Tabela 3), persistiram associadas a risco aumentado de infecção as seguintes variáveis: idade maior ou igual a 60 anos $(\mathrm{RP}=2,2$; IC-95\% $=$ $1,2-4,6)$, obesidade $(\mathrm{RP}=3,0 ; \mathrm{IC}-95 \%=1,9-4,8)$, diabetes $(\mathrm{RP}=5,3$; IC-95\% $=3,2-8,9)$ e tempo cirúrgico maior que 2 horas $(\mathrm{RP}=2,2$; IC-95\% $=$ 1,3-3,4). A antibioticoprofilaxia persistiu associada a menor risco de infecção ( $\mathrm{RP}=0,5$; IC-95\% $=0,4-0,7)$. 
Tabela 2 - Risco de infecção do sítio cirúrgico de acordo com vários parâmetros epidemiológicos clínicos, e com o uso ou não de antibioticoprofilaxia.

\begin{tabular}{|c|c|c|c|c|c|}
\hline \multicolumn{2}{|c|}{ Parâmetros } & \multirow{2}{*}{$\begin{array}{c}\begin{array}{c}\text { Com } \\
\text { infecção }\end{array} \\
35\end{array}$} & \multirow{2}{*}{$\begin{array}{c}\begin{array}{c}\text { Sem } \\
\text { infecção }\end{array} \\
347\end{array}$} & \multirow{2}{*}{$\begin{array}{l}\mathbf{R P} \\
1,0\end{array}$} & \multirow[t]{2}{*}{ IC a $95 \%$} \\
\hline Idade & $<60$ anos & & & & \\
\hline \multirow[t]{2}{*}{ Obesidade } & Não & 23 & 306 & 1,0 & \\
\hline & Sim & 19 & 66 & 3,2 & $1,8-5,6$ \\
\hline Diabetes & Presente & 09 & 09 & 6,0 & $3,4-10,6$ \\
\hline \multirow[t]{2}{*}{ Hipertensão } & Ausente & 31 & 308 & 1,0 & \\
\hline & Presente & 11 & 64 & 1,6 & $0,8-3,0$ \\
\hline Indicação cirúrgica & Doença benigna & 40 & 356 & 1,0 & \\
\hline \multirow[t]{2}{*}{ Tipo de incisão } & Mediana & 09 & 86 & 1,0 & \\
\hline & Pfannenstiel & 33 & 286 & 0,9 & $0,5-1,9$ \\
\hline \multirow[t]{2}{*}{ Duração da cirurgia } & $\leq 2$ horas & 27 & 308 & 1,0 & \\
\hline & $>2$ horas & 15 & 64 & 2,4 & $1,3-4,2$ \\
\hline
\end{tabular}

Obesidade = IMC 27,5; HA máx $>140 \mathrm{mmHg}$; mín $>90 \mathrm{mmHg}$

$\mathrm{RP}=$ Risco de prevalência; $I C=$ intervalo de confiança

Tabela 3 - Risco ajustado de infecção em pacientes submetidas a histerectomia total abdominal no IMIP (regressão logística múltipla).

\begin{tabular}{lcc}
\hline Variável & $\begin{array}{c}\text { Risco de prevalência } \\
\text { ajustado }\end{array}$ & IC a 95\% \\
\hline Idade $\geq 60$ anos & 2,2 & $1,2-4,6$ \\
Obesidade & 3,0 & $1,9-4,8$ \\
Tempo cirúrgico >2 horas & 2,2 & $1,3-3,4$ \\
Diabetes & 5,3 & $3,2-8,9$ \\
Antibioticoterapia profilática & 0,5 & $0,4-0,7$ \\
\hline
\end{tabular}

\section{Discussão}

De acordo com os resultados do presente estudo, os fatores associados a risco aumentado de infecção do sítio cirúrgico foram: idade maior ou igual a 60 anos, obesidade, diabetes e tempo cirúrgico prolongado (maior que 2 horas). A antibioticoprofilaxia, por outro lado, determinou significativa redução do risco de infecção, em torno de $60 \%$.

Esses resultados corroboram os de diversos estudos, que apontam a idade avançada, a obesidade e o diabetes como os mais importantes fatores de risco para infecção pós-operatória, não apenas em ginecologia como em cirurgia geral e outras especialidades ${ }^{3,5-7,12}$.
Considerando-se a idade, taxas progressivamente maiores de infecção têm sido apontadas em grupos etários a partir dos 50 anos $^{1}$. Atribui-se o aumento do risco de infecção tanto à maior freqüência de doenças clínicas (em especial o diabetes) e depressão imunológica nos idosos, como também à maior freqüência de intervenções por neoplasia. No entanto, uma maior freqüência de infecções pós-histerectomia tem sido descrita para pacientes jovens, o que se atribui à presença de uma flora vaginal mais virulenta ${ }^{1}$. Em nosso estudo, a freqüência de infecção foi mais de 2 vezes maior quando a idade era igual ou superior a 60 anos e não se observou risco aumentado para pacientes jovens.

Por outro lado, tanto diabetes como obesidade são citados como fatores de risco para infecção pós-operatória na maioria dos trabalhos publicados na literatura científica internacional ${ }^{1,3,5-7,12}$. Pacientes com diabetes apresentam defeitos na quimiotaxia, aderência e fagocitose dos granulócitos, que se tornam menos aptos à defesa contra infecções fúngicas e bacterianas. Em nosso estudo, a maioria das pacientes diabéticas eram tipo II, e a cirurgia só foi realizada com glicemia abaixo de $180 \mathrm{mg} \%$, monitorizandose o controle glicêmico com hemoglicoteste e administração de insulina simples no pré e pósoperatório imediato. Mesmo com esse adequado controle metabólico, o risco de infecção foi 6 
vezes maior na presença de diabetes.

A obesidade per se constitui também fator de risco independente para infecção cirúrgica, existindo inclusive relatos de aumento da freqüência de infecção à medida que aumenta a espessura do tecido celular subcutâneo ${ }^{12}$. As alterações nutricionais podem explicar a má cicatrização, porém a obesidade também se associa a outros fatores de risco, como diabetes e tempo cirúrgico prolongado, bem como à má higiene ${ }^{1}$. O risco de prevalência de infecção em obesas neste estudo foi de 3,2, comparável às taxas referidas por outros autores ${ }^{1,5,12}$.

A associação entre tempo cirúrgico prolongado e infecção pós-operatória também foi descrita em diversos estudos, possivelmente pelo aumento do potencial de contaminação do campo cirúrgico. Além disso, o prolongamento do tempo cirúrgico pode estar associado a dificuldades técnicas em casos de maior complexidade, a cirurgia por doenças malignas, à obesidade e à inexperiência do cirurgião, todos esses fatores independentes de risco para infecção ${ }^{1}$. A média de duração das histerectomias em nosso serviço foi de 116 minutos, e verificou-se que a duração da cirurgia além de 2 horas associouse a um risco 2 vezes maior de infecção pós-operatória. Lembramos que essas cirurgias em nosso serviço são sempre realizadas por médicosresidentes (em treinamento), porém a freqüência de tempo cirúrgico superior a 2 horas foi de apenas $19 \%$, e a taxa de infecção pós-operatória, em torno de $10 \%$, comparável às descritas em publicações diversas.

Ao contrário do que tem sido descrito por outros autores ${ }^{1}$, a presença de doença maligna como indicação da cirurgia não esteve associada a aumento do risco de infecção, o que provavelmente se explica pelo pequeno número de pacientes com neoplasia maligna (18 casos) em nossa casuística e pela exclusão das cirurgias radicais (Wertheim-Meigs) do estudo.

A questão da antibioticoprofilaxia nas histerectomias abdominais é ainda controversa, uma vez que sua efetividade tem sido questionada ${ }^{1,7,8,11}$. Apesar de alguns estudos documentarem redução do risco de infecção, outros apontam um beneficio restrito às situações de risco (obesidade, diabetes etc.). Descreve-se uma menor eficácia para a histerectomia abdominal em relação à vaginal, na qual as vantagens da antibioticoprofilaxia são indiscutiveis ${ }^{1}$. Recomenda-se portanto que a indicação de antibioticoterapia profilática seja individualizada de serviço para serviço, devendo ser adotada sempre que a taxa de infecção do sítio cirúrgico for superior a $10 \%{ }^{1}$. Em nossa casuística, a freqüência de in- fecção foi em torno de $20 \%$ no grupo ao qual não se administrou antibiótico, documentando-se uma redução de $60 \%$ no risco quando se realizou antibioticoprofilaxia, o que justifica portanto sua adoção sistemática em nosso serviço.

Outros fatores de risco que têm sido exaustivamente estudados são as infecções vaginais, especialmente a vaginose bacteriana e a tricomoniase ${ }^{10}$, que não foram no entanto variáveis pesquisadas em nosso estudo, até porque sua presença constitui contra-indicação à cirurgia eletiva em nosso serviço, e todas as pacientes que porventura tivessem esse diagnóstico foram adequadamente tratadas antes da realização da histerectomia. A condição econômica desfavorável, que também representa risco aumentado de infecção pós-operatória ${ }^{1}$, constituiu uma constante em nosso estudo, uma vez que todas as pacientes do IMIP fazem parte da clientela do SUS (Sistema Único de Saúde), pressupondo-se uma certa uniformidade de nível socioeconômico. Acreditamos que a escolaridade poderia ter sido utilizada como critério para identificar possiveis diferenças na população estudada, porém não foi possível pesquisar esta variável, já que não constava em todos os prontuários.

$\mathrm{Na}$ análise de regressão logística múltipla, todos os fatores que tiveram associação significativa com a infecção do sítio cirúrgico na análise bivariada persistiram com um risco de prevalência semelhante, com aumento do risco para a idade maior que 60 anos, obesidade, diabetes e tempo cirúrgico prolongado, e redução do risco quando se realizou antibioticoprofilaxia, o que indica que apesar de possiveis inter-relações entre todas essas variáveis, elas representam fatores de risco independentes para infecção póshisterectomia. Apesar de o caráter retrospectivo do presente estudo não ter permitido a inclusão no modelo de outras variáveis potencialmente confundidoras, acreditamos que as conclusões aqui obtidas são válidas e encontram-se de acordo com a maioria das publicações relacionadas ao assunto.

Embora a maioria dos fatores de risco encontrados no presente estudo seja dificil de controlar ou prevenir (como idade, obesidade e diabetes), a identificação de qualquer destes deve constituir indicação obrigatória de antibioticoprofilaxia e acompanhamento rigoroso no pós-operatório, visando à identificação e tratamento adequado da infecção do sítio cirúrgico, conforme as normas do $\mathrm{CDC}^{9}$. A realização de estudos longitudinais (coorte) é necessária no sentido de manter vigilância constante e identificar possiveis mudanças no perfil epidemiológico das in- 
fecções pós-histerectomia, principalmente depois da normatização do uso de antibioticoterapia profilática.

\section{SUMMARY}

Purpose: to determine the main factors associated with the occurrence of surgical site infection in patients submitted to total abdominal hysterectomy at the Instituto Materno - Infantil de Pernambuco (iMIP).

Methods: a cross-sectional study was conducted, enrolling patients submitted to total abdominal hysterectomy at IMIP who returned to postsurgical consultation for infection control, between January, 1995 and December, 1998 ( $n=$ 414). The frequency of surgical site infection (defined according to the CDC criteria, 1998) was 10\% (42 cases). Prevalence risk (PR) of infection (dependent variable) and its $95 \%$ confidence interval (CI) were calculated for independent variables: age, obesity, hypertension, diabetes, malignant pathology, type of incision, duration of surgery and antibiotic prophylaxis. Multiple logistic regression analysis was used to determine adjusted risk of infection.

Results: a significantly increased risk of infection was found for the following variables: age $\geq 60$ years $(P R=$ 2.39, $95 \% C I=1.15-4.94)$, obesity $(P R=3.2,95 \% C I=$ 1.83-5.59), duration of surgery $>2$ hours $(P R=2.35,95 \%$ $C I=1.32-4.21)$ and diabetes $(P R=6.0,95 \% C I=3.41$ 10.57). On the other hand, risk of infection was significantly reduced when antibiotic prophylaxis was administered $(P R=0.38,95 \% C I=0.21-0.68)$. Type of incision, malignant disease and hypertension were not associated with infection.

Conclusions: the factors associated with increased risk of surgical site infection after total abdominal hysterectomy at IMIP were: age $>60$ years, obesity, diabetes and surgical duration $>2$ hours. Antibiotic prophylaxis showed a protective effect with reduction of risk of infection.

KEY-WORDS: Hysterectomy. Infection. Postoperative complications.

\section{Referências}

1. Thompson JD, Warshaw JS. Hysterectomy. In: Rock JA, Thompson JD, editors. Te Linde's Operative Gynecology. $8^{\text {th }}$ ed. Philadelphia: LippincottRaven; 1997. p.771-854.

2. Wilcox L, Koonin L, Pokras R, Strauss LT, Xia Z, Peterson HB. Hysterectomy in the United States, 1988-1990. Obstet Gynecol 1994; 83:549-55.

3. Harris WJ. Early complications of abdominal and vaginal hysterectomy. Obstet Gynecol Surv 1995; 50:795-805.

4. Harris WJ. Complications of hysterectomy. Clin Obstet Gynecol 1997; 40:928-38.

5. Hager WD. Postoperative infections: prevention and management. In: Rock JA, Thompson JD, editors. Te Linde's Operative Gynecology. $8^{\text {th }}$ ed. Philadelphia: Lippincott-Raven; 1997. p.233-44.

6. Kandula PV, Wenzel RP. Postoperative wound infection after total abdominal hysterectomy: a controlled study of the increased duration of hospital stay and trends in postoperative wound infection. Am J Infect Control 1993; 21:201-4.

7. Persson E, Bergström M, Larsson PG, et al. Infections after hysterectomy. A prospective nation-wide Swedish study. The Study Group on Infectious Diseases in Obstetrics and Gynecology within the Swedish Society of Obstetrics and Gynecology. Acta Obstet Gynecol Scand 1996; 75:757-61.

8. Taylor G, Herrick T, Mah M. Wound infections after hysterectomy: opportunities for practice improvement. Am J Infect Control 1998; 26:2547.

9. Centers for Disease Control. Draft guideline for the Prevention of surgical site infections, 1998. Fed Regist 1998; 63:33168-92.

10.Soper DE, Bump RC, Hurt WG. Bacterial vaginosis and trichomoniasis vaginitis are risk factors for cuff cellulitis after abdominal hysterectomy. Am J Obstet Gynecol 1990; 163:1016-23.

11.Hemsell DL, Johnson ER, Hemsell PG, Nobles BJ, Heard MC. Cefazolin for hysterectomy prophylaxis. Obstet Gynecol 1990; 76:603-6.

12.Soper DE, Bump RC, Hurt WG. Wound infection after abdominal hysterectomy: effect of the depth of subcutaneous tissue. Am J Obstet Gynecol 1995; 173: 465-71. 